\title{
SAR Imaging for Ships in Rough Seas Based on Deconvolutive STFT Spectrogram Method
}

\author{
Zhao Yu, Yang Beibei, Sun Jinping, Zhang Yuan \\ School of Electronic and Information Engineering \\ Beihang University \\ Beijing, China \\ sunjinping@buaa.edu.cn
}

\begin{abstract}
There is multi-degree-of-freedom non-cooperation motion of ships in rough seas which makes the quality of the ships' SAR images get poor. In this paper, the ship motion model was built based on the strip theory at first. Then, the quality of the ships' SAR images which are in different sea states was analyzed using the Standard Chirp Scaling Algorithm (CSA). At last, we propose a new SAR imaging method which is based on Deconvolutive Short-Time Fourier Transform ( DSTFT) Spectrogram. Through simulation, the validity of the proposed method to improve the quality of SAR imaging was verified.
\end{abstract}

Index Terms-SAR imaging, deconvolutive short-time Fourier transform spectrogram, ship motion modeling.

\section{INTRODUCTION}

Getting high-resolution images of ship targets on the sea and recognizing them based on these images have great value in both military and civilian fields. Synthetic aperture radar can obtain high-resolution images of ship targets in long range and all weather conditions, which has obvious advantages compared with other imaging tools ${ }^{[1]}$. Due to the effect of the waves, the moving ship which is on the sea has complex motion form. Therefore, the SAR imaging gets difficult because that it is hard to estimate the motion law of the ship's scattering point in three-dimensional space.

When ships move heavily, traditional SAR imaging methods which require correction and compensation may make the images get blurry. However, using time-frequency analysis can improve the image quality in this condition ${ }^{[2]}$. Both range and azimuth range signal of the SAR echo can be approximated as LFM signal that is a typical non-stationary signal, and time-frequency analysis is a powerful tool for analyzing non-stationary signals. The common used timefrequency distributions include Short-Time Fourier Transform (STFT), Winger-ville distribution (WVD), etc. Whereas, there are some disadvantages in both of them. STFT can't simultaneously obtain both high time resolution and frequency resolution. And, WVD has cross-term.

To begin with, this paper builds the ship motion model in rough seas using the strip theory ${ }^{[3]}$. In addition, it analyzes the quality of ships' SAR images by the standard CSA in different sea states. At last, this paper proposes a new SAR imaging method that is based on DSTFT spectrogram. DSTFT spectrogram has a better time-frequency resolution and crossterm suppression performance compared with some traditional methods. Through simulation, the method's validity in improving the quality of SAR imaging is demonstrated

\section{SHIP MOVING MODEL}

When ships move on the sea there exist multiple degree of freedom's complex motion owing to the influence of ship' propulsion and control, wind and waves. Random waves acting on the hull of the ship lead to the disturbing forces and disturbance torques, then the six-degree-of-freedom motion model of the ship can be obtained with the forces and torques as the input parameters of ship's motion equation ${ }^{[4]}$.

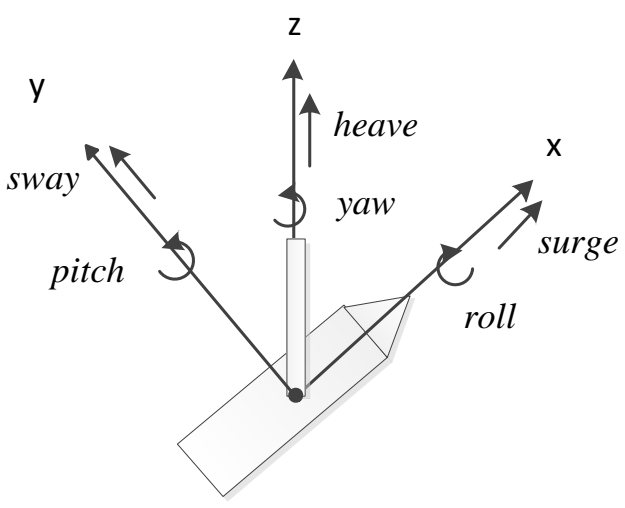

Fig. 1. Reference system of the ship

As is shown in Fig. 1, the complex motion of the ship can be described by six degrees of freedom. Surge, sway and heave are the translational motion along the three axes respectively and roll, yaw and pitch are the rotational motion with the three axes as rotational shafts. The motions of the six degrees are interrelated, however within linear theory, using the ship symmetry and considering that the surge motion and the power-drive translational motion can be dealt with together, the ship motion equation of six degrees can be divided into two sets of coupling equations as follows ${ }^{[5]}$.

1) Heave-Pitch Motions

$$
\left\{\begin{array}{l}
\left(A_{33}+m\right) \ddot{z}+B_{33} \dot{z}+C_{33} z+A_{35} \ddot{\theta}+B_{35} \dot{\theta}+C_{35} \theta=F_{3}(t) \\
A_{53} \ddot{z}+B_{53} \dot{z}+C_{53} z+\left(A_{55}+I_{y}\right) \ddot{\theta}+B_{55} \dot{\theta}+C_{55} \theta=F_{5}(t)
\end{array}\right.
$$

2) Sway-Roll-Yaw Motions 


$$
\left\{\begin{array}{l}
\left(m+A_{22}\right) \ddot{y}+B_{22} \dot{y}+A_{24} \ddot{\varphi}+A_{24} \dot{\varphi}+A_{26} \ddot{\psi}+B_{26} \dot{\psi}=F_{2}(t) \\
A_{42} \ddot{y}+B_{42} \dot{y}+\left(I_{x}+A_{44}\right) \ddot{\varphi}+B_{44} \dot{\varphi}+C_{44} \varphi+A_{46} \ddot{\psi}+B_{46} \dot{\psi}=F_{4}(t) \\
A_{62} \ddot{y}+B_{62} \dot{y}+A_{64} \ddot{\varphi}+B_{64} \dot{\varphi}+\left(I_{z}+A_{66}\right) \ddot{\psi}+B_{66} \dot{\psi}=F_{6}(t)
\end{array}\right.
$$

where $y$ and $z$ are the sway and heave displacement, $\theta, \varphi$ and $\psi$ are the pitch, roll and yaw angles; $m$ is the mass of the ship, $I_{x}, I_{y}$ and $I_{z}$ are the mass moment of inertia which are around $x, y$ and $z ; \quad A_{i j}, B_{i j}$ and $C_{i j}(i, j=2,3,4,5,6)$ are the added mass, damping, hydrostatic coefficient matrices, respecttively; $F_{i}(i=2,3,4,5,6)$ is the exciting forces.

The wave is considered as an ergodicity stationary random process, and the ship can be regarded as a linear time-invariant system in certain sea condition, speed, and course. So, the wave height, the disturbing forces and disturbance torques of the ship can be generated using the theory of random signal analysis. At first, when the white gauss noise as the input, the output of the linear system is the simulation wave with encounter wave spectrum $S(\omega)^{[4]}$. Then, the wave height drives the linear transfer functions $G_{j}(\omega)$ to obtain the final disturbing forces and disturbance torques of each degree of freedom. In this paper, the wave spectrum is chosen as PiersonMoscowitz spectrum ${ }^{[6]}$. In summary, the process of ship motion modeling can be described as Fig. 2.

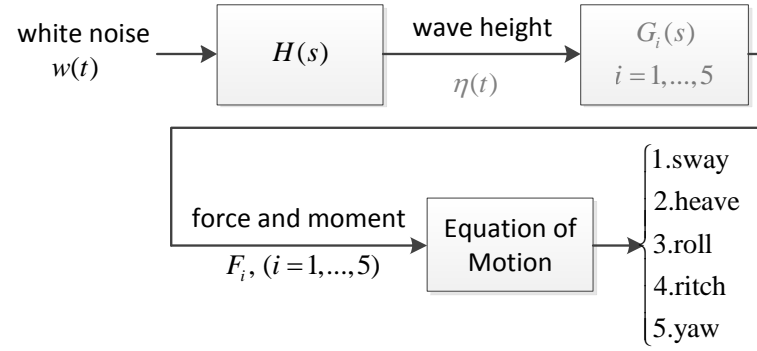

Fig. 2. Process of ship motion modeling

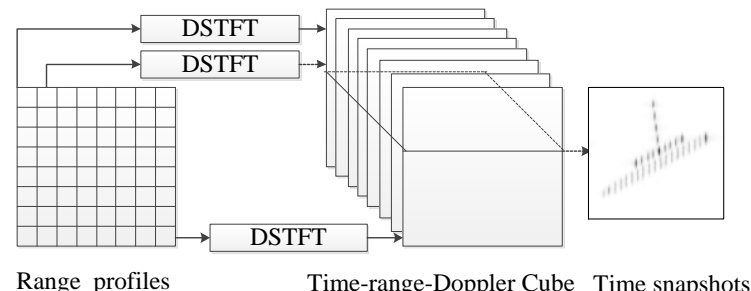

Fig. 3. DSTFT spectrogram method diagram

\section{SAR IMAGE COMPENSATION BASED ON DSTFT SPECTROGRAM METHOD}

SAR imaging is based on range-Doppler principle, in general, the target is assumed stationary. But, the ship target in rough seas has complex motion, as stated in above. Thus, the SAR imaging needs compensation. In this paper the SAR images is compensated by DSTFT spectrogram method.
DSTFT spectrogram method gets the time-frequency distribution of the echo and then merges the time-frequency analysis results of each range bin together to obtain the target's instantaneous image. The process of DSTFT spectrogram method is shown in Fig. 3.

\section{Deconvolutive ShorT-Time Fourier Transform SPECTROGRAM}

The STFT of the signal $s(t)$ is defined as

$$
\operatorname{STFT}_{s}(t, f)=\int_{-\infty}^{+\infty} s(\tau) h^{*}(\tau-t) e^{-j 2 \pi f \tau} d \tau
$$

where $h(u)$ is the window function, $*$ denotes conjugate transpose. STFT can't achieve both high time resolution and high frequency resolution simultaneously as a result of windowing operation. But, there is no cross-term in the STFT spectrogram, which is defined as

$$
\operatorname{SPEC}_{s}(t, f)=\left|\int_{-\infty}^{+\infty} s(\tau) h^{*}(\tau-t) e^{-j 2 \pi f \tau} d \tau\right|^{2}
$$

For a signal $s(t)=s_{1}(t)+s_{2}(t)$, it can be proved that

$$
\begin{array}{r}
\operatorname{STFT}_{s}(t, f)=\operatorname{STFT}_{s_{1}}(t, f)+\operatorname{STFT}_{s_{2}}(t, f) \\
\operatorname{SPEC}_{s}(t, f)=\operatorname{SPEC}_{s_{1}}(t, f)+\operatorname{SPEC}_{s_{2}}(t, f) \\
+2 \operatorname{Re}\left\{\operatorname{SPEC}_{s_{1}}(t, f) \times \operatorname{SPEC}_{s_{2}}^{*}(t, f)\right\}
\end{array}
$$

In order to obtain a good resolution in both the time and frequency domains at a same time, WVD has been widely used and its definition is

$$
\operatorname{WVD}(t, f)=\int_{-\infty}^{+\infty} s\left(t+\frac{\tau}{2}\right) s^{*}\left(t-\frac{\tau}{2}\right) e^{-j 2 \pi f \tau} d \tau
$$

In contrast to the linear representation of STFT, the WVD is quadratic. The drawback of WVD is that there is inevitable cross-term for multi-component signals. The WVD of signal $s(t)=s_{1}(t)+s_{2}(t)$ is

$$
\begin{aligned}
\operatorname{WVD}_{s}(t, f) & =\operatorname{WVD}_{s_{1}}(t, f)+\operatorname{WVD}_{s_{2}}(t, f) \\
& +2 \operatorname{Re}\left\{\operatorname{WVD}_{s_{1}, s_{2}}(t, f)\right\}
\end{aligned}
$$

The following relationship has been proven in [7]

$$
\begin{array}{r}
\operatorname{SPEC}_{s}(t, f)=\int_{-\infty}^{+\infty} \int_{-\infty}^{+\infty}\left(\mathrm{WVD}_{h}(u, v)\right. \\
\left.\times \mathrm{WVD}_{s}(t-u, f-v)\right) d u d v
\end{array}
$$

Where $\mathrm{WVD}_{x}(t, f)$ and $\mathrm{WVD}_{h}(u, f)$ are the WVDs of the signal $x(t)$ and the window function $h(u)$. To describe it simply, we rewrite the eq.(9) as

$$
P_{s}=\mathbf{W}_{s} \otimes_{t-f} \mathbf{W}_{h}
$$

where $P_{s}$ is the STFT spectrogram of the signal, $\mathbf{W}_{s}$ is the WVD of the signal, $\mathbf{W}_{h}$ is the WVD of the window function and $\otimes_{t-f}$ denotes 2-D convolution.

According to eq. (10), the signal's STFT spectrogram is obtained from 2-D convolution between the signal's WVD and the window function's WVD. Considering that the STFT spectrogram has almost no cross-term, which reduces the performance of the time-frequency analysis for multi- 
component signals, so if $\hat{\mathbf{W}}_{s}$ the estimate of $\mathbf{W}_{s}$ can be calculated by $P_{s}$ and $\mathbf{W}_{h}$, then $\hat{\mathbf{W}}_{s}$ will have fine timefrequency resolution close to the WVD but without cross-term, which cannot be avoided in conventional WVD. DSTFT spectrogram method calculates $\hat{\mathbf{W}}_{s}$ using $P_{s}$ and $\mathbf{W}_{h}$ through deconvolution algorithm based on eq. (10).

Deconvolution has been widely used in image restoration technology, There are a lot of algorithms available, such as Lucy-Richardson (L-R)algorithm, maximum entropy (ME) algorithm and Gerchberg-Saxton (G-S) algorithm, etc. In this paper, we adopt L-R algorithm ${ }^{[8,9]}$ in the deconvolution process for (10) and the iterative calculation process can be described as ${ }^{[10]}$

$$
\hat{\mathbf{W}}_{s}(k)=\hat{\mathbf{W}}_{s}(k-1)\left(\mathbf{W}_{h} * \frac{P_{s}}{\mathbf{W}_{h} \otimes_{t-f} \hat{\mathbf{W}}_{s}(k-1)}\right)
$$

Where $k$ is the current iteration number, $*$ is the correlation operator, and the iterative initial value is set as $\hat{\mathbf{W}}_{s}(0)=P_{s}$. In general, the deconvolutive process will converge to a desired result within ten iterations.

\section{Simulation RESUlts}

This paper adopts the particulars of the DD963 destroyer provided by MIT and works out the simulation result of the destroyer's heave, pitch, sway, roll, and yaw motions with the ship motion model as described above. The simulation results of sea state 5 are shown in Fig. 4-8. Where, the conditions of heave-pitch motions are fellows: significant wave height is $10 \mathrm{ft}$ $(3.05 \mathrm{~m})$, ship speed is $21 \mathrm{ft} / \mathrm{s}(6.40 \mathrm{~m} / \mathrm{s})$, angle between heading and wind direction is $0^{\circ}$; the conditions of sway-roll-yaw motions are fellows: significant wave height is $10 \mathrm{ft}(3.05 \mathrm{~m})$, ship speed is $15.5 \mathrm{ft} / \mathrm{s}(4.72 \mathrm{~m} / \mathrm{s})$, angle between heading and wind direction is $45^{\circ}$.

Then, this paper analyzes the influence of the complex motion of ship on SAR imaging using the ship motion model of DD693. It is assumed that the ship is composed of ideal scattering points which is depicted in Fig. 9

LFM signal is chosen as the emission signal, and the standard CSA algorithm is used in imaging. The specific parameters of the radar are listed in Table 1

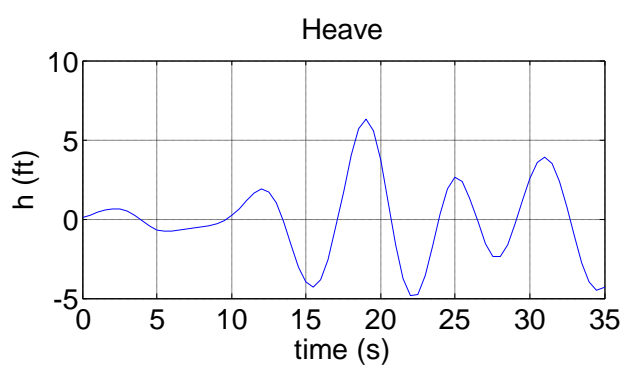

Fig. 4. Simulation results of heave motion

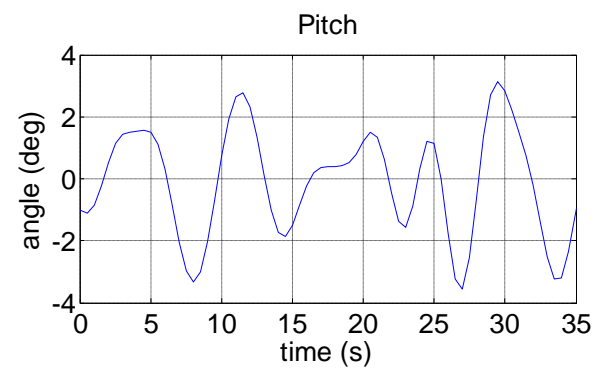

Fig. 5. Simulation results of pitch motion

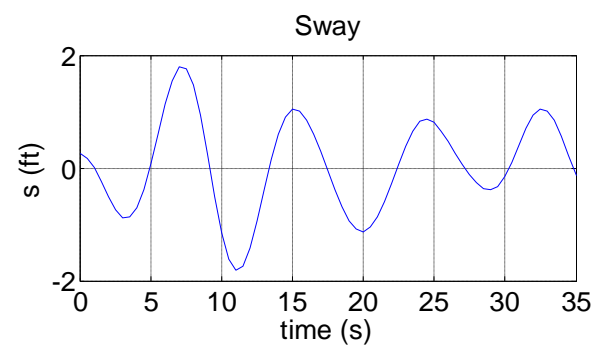

Fig. 6. Simulation results of sway motion

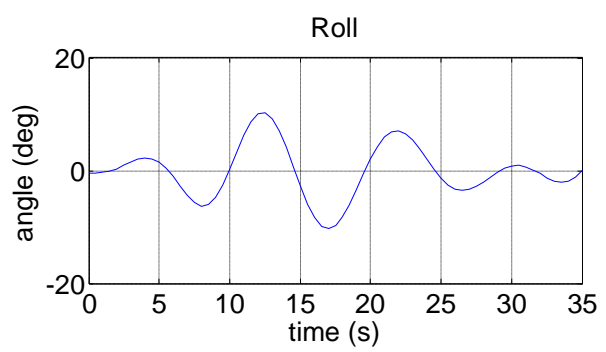

Fig. 7. Simulation results of roll motion

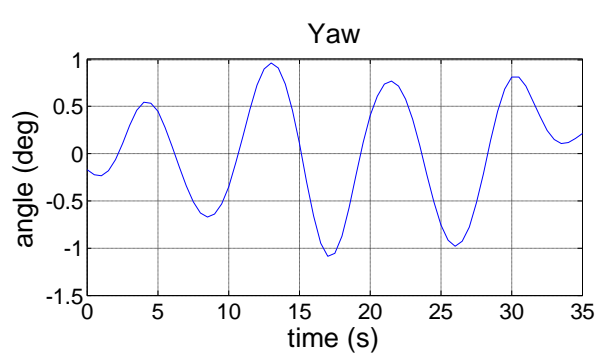

Fig. 8. Simulation results of yaw motion

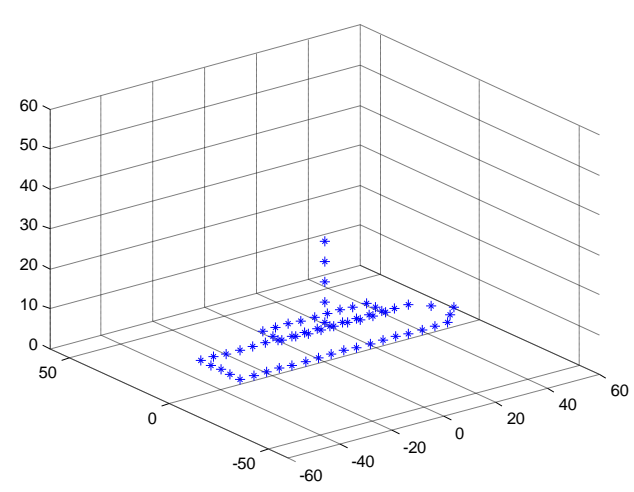

Fig. 9. Schematic diagram of the ship target

We select the ship targets in sea state 3 and 5 respectively for SAR imaging so as to analyze the influence of different sea states on SAR imaging. The results are as follows: 
TABLE I. PARAMETERS OF SAR

\begin{tabular}{|c|c|}
\hline Parameter Name & Value \\
\hline wave length $\lambda$ & $0.032 \mathrm{~m}$ \\
\hline bandwidth $B_{r}$ & $150 \mathrm{MHz}$ \\
\hline pulse width $T_{v}$ & $16 \mu \mathrm{s}$ \\
\hline Range revolution & $1 \mathrm{~m}$ \\
\hline Cross range revolution & $1 \mathrm{~m}$ \\
\hline Speed of platform $V$ & $200 \mathrm{~m} / \mathrm{s}$ \\
\hline
\end{tabular}

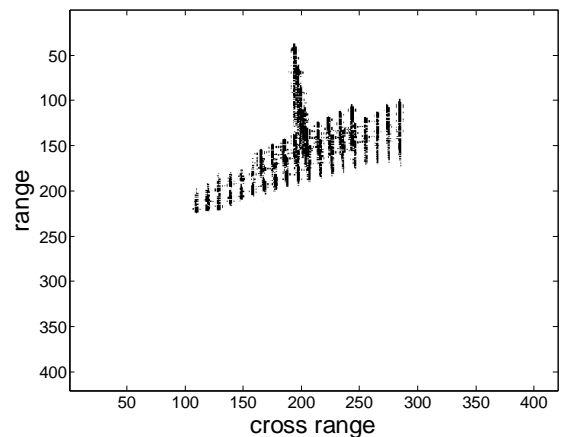

Fig. 10. SAR image in sea state 3 (significant wave height: $3.05 \mathrm{ft}(1 \mathrm{~m})$ )

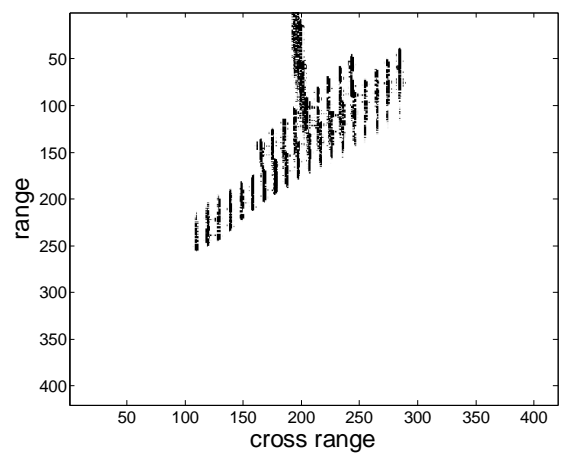

Fig. 11. SAR image in sea state 5 (significant wave height: $10 \mathrm{ft}(3.05 \mathrm{~m})$ )

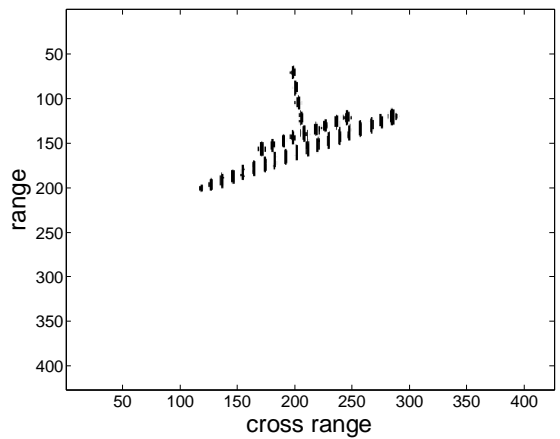

Fig. 12. SAR image using DSTFT spectrogram method in sea state 5

Comparing Fig.10 with Fig.11, It can be concluded that the quality of the SAR imaging will become worse in higher sea scale because that the amplitude of the non-cooperation movement get bigger when the sea scale get higher.
In order to get a distinct image, we apply DSTFT spectrogram method to processing the SAR echo of the ship target in sea state 5 . The result is given in Fig. 12, which shows that the quality of the image enhances evidently.

\section{CONCLUSION}

The ship has complex motion form in rough seas and it can be considered as periodical rocking and oscillatory motion though modeling analysis. The non-cooperation motion cannot avoid making the conventional SAR imaging method have performance degradation. The non-cooperation movement has impact on the quality of the SAR imaging result. This paper has proposed the DSTFT spectrogram method for SAR imaging. And simulation results have shown the effectiveness of this method.

\section{ACKNOWLEDGMENT}

This work was supported by the National Basic Research Program of China (2010CB731903).

\section{REFERENCES}

[1] Ausherman A., Kozma A. and Waker J. L., "Developments in radar imaging," IEEE Transactions on Aerospace Electronic Systems, pp. 363-400, Apr 1984.

[2] VC.Chen and HaoLing, Time-Frqeueney Transforms for Radar Imaging and Signal Analysis, London: Arteeh HouseInc, 2002

[3] J. H. Milgram, "Strip theory for underwater vehicles in water of finite depth", Engineering Mathematics, V58 (1-4), pp. 31-50 2007.

[4] M. Triantafyllou, M. bodsoon and M. athans, "Real time estimation of ship motions using Kalman filtering techniques," IEEE journal of oceanic engineering, vol.OE-8, No.1, January 1983.

[5] N.Salvesen, E. O. Tuck, and O. Faltinsen, "Ship motions and sea loads," Trans. SNAME, vol. 78, 1970.

[6] M. Triantafyllou and M.Bodson, "Real time estimation of the heaving and pitching motions of a ship using a Kalman filter," Proc. Oceans 81 Boston MA, pp.1090-1095, Sept 1981.

[7] W. Lu and Q. Zhang, "Deconvolutive short-time Fourier transform spectrogram", IEEE Signal Processing Letters, vol.16, no.7, pp.576-579, 2009.

[8] W. H. Richardson, "Bayesian-based iterative method of image restoration", J. Opt. Soci. America, vol.62, no.1, pp.55-59, 1972.

[9] L. B. Lucy, "An iterative technique for the rectification of observed distributions", Astron. J., vol. 79, no. 6, pp.745-754, 1974.

[10] D. S. C. Biggs and M. Andrews, "Acceleration of iterative image restoration algorithms", Appl. Opt., vol. 36, no. 8, pp.1766-1775, 1997. 